\title{
Alert Routing In Wireless Video Sensor Networks for Monitoring Applications
}

\author{
Youssef Benabbassi \\ Bechar University, \\ Bechar, Algeria
}

\author{
Hafid Haffaf \\ Oran University, \\ Oran, Algeria
}

\author{
Congduc Pham \\ Pau University, \\ Pau, France
}

\begin{abstract}
Wireless sensor networks are appropriate monitoring tools used in surveillance applications. Organizations all over the world are realizing the effectiveness of protecting people, places and things with advanced video surveillance systems to increase safety. Wireless infrastructure allows them to deploy and extend video surveillance capability in virtually any indoor or outdoor environment. The network lifetime is directly related to the energy resources of the sensor nodes and can be extended by energy-aware protocols. The LIUPPA, France Laboratory has proposed a wireless video sensors model dedicated to intrusion detection surveillance application. Our aim is to extend this work by new algorithms that optimize the alert propagation message diffusion.
\end{abstract}

The main idea of our algorithm is to reduce the flooding of the message alert by propagating them only for the list of the sensors located in the field of view (FOV) of the sensor alerted. This reduces considerably the effects of implosion. In this paper, we describe the WVSN model proposed by the LIUPPA. Moreover, we explained and discussed the result of our algorithm under Omnetpp/Castalia Simulator.

Keywords: Wireless Sensor Networks; Routing; Surveillance Application, Wireless Video Sensor Networks; Energy Consumption.

\section{INTRODUCTION}

In the past, the wired sensor networks were used in the factory control and automation system. An exponential growth in wireless communication techniques has made it possible for wireless control and automation system [1].

Rapid advances in Micro-Electro-Mechanical Systems (MEMS) technology have made possible the existence of tiny sensor nodes equipped with sensing, communication and processing capabilities. When sprayed in an area, they are capable of forming a multi-hop wireless network known as Wireless Sensor Network (WSN)[2]. The availability of lowcost hardware is enabling the development of wireless multimedia sensor networks (WMSNs), i.e., networks of resource-constrained wireless devices that can retrieve multimedia content such as video and audio streams, still images, and scalar sensor data from the environment [3]. Wireless Video Sensor Networks (WVSNs) are a category of WSNs in which sensor nodes are equipped with a digital camera.

Wireless sensor networks are appropriate tools to monitor an area for surveillance. Video surveillance is being implemented on an unprecedented scale. Public safety agencies, municipalities, maritime ports, transit authorities and other government agencies depend on video surveillance to protect their constituents and property. For law enforcement, video surveillance is essential to deter crime, improve incident response time and provide forensic data. Video surveillance is also essential for organizations that have infrastructures spread over large areas, such as public transportation, university and corporate campuses, retail, construction, logistics, mining, oil and gas [4].
WVSNs suffer from the same problems as WSNs such as application dependency, energy constraints, high sensor density, limited bandwidth and dynamic topology. Terrance Boult and al. in [5] reviews significant issues, design constraints and accomplishments of work on multi-camera sensor networks combining multiple omni-directional imaging sensors, traditional stationary cameras and pan-tilt sensors. To analyze the surveillance performance of the network, the author in [6] proposes deployment quality measures and a trade-off between the number of sensors and the deployment quality have been discussed.

The performance of a surveillance wireless sensor network is generally measured with its detection capability within a monitored zone. Can Komar and al. provide a tool to the network to derive the expected detection performance and risk analysis framework for a given sensor network with realistic border surveillance scenario parameters [7]. Antonio-Javier and al. introduces that a video-surveillance is a solution to detect and identify intruders as well as to better take care of a process [8]. They propose a new platform called integrated wireless sensor network solution for precision agriculture. Congduc Pham and al. from the LIUPPA[19], France Laboratory; propose a multiple-level activity model that uses behavior functions to define application classes and allows for adaptive scheduling based on the criticality application of multiple cover sets per sensor node [9]. Our aim is to extend this application with an algorithm proposed to manage the alert propagated message diffusion. The main idea of our algorithm is to reduce the flooding of the message alert in order to reduce energy consuming.

The paper is organized as follows. Section 2 presents the related works in wireless routing protocols. Section 3 presents 
the WVSN model developed at the LIUPPA France Laboratory which is the main stone of our approach. Section 4 presents our algorithm proposed to manage the diffusion of the message alert through the network taking to reduce considerably the effects of implosion. Section 5 presents the simulation results under the Omnetpp/Castalia Simulator. Finally, section 6 concludes the paper and points out open research problems.

\section{ROUTING PROTOCOLS IN WSN}

Routing protocols in WSN are topical issue in this domain and are covered by a rich literature researchs. Kemal Akaya and al. in [10] surveys recent routing protocols for sensor networks and presents a classification for various approaches pursued also each routing protocol is described and discussed. Ian F.Akyilidiz in [11] surveyed the state of the art in algorithms, protocols, and hardware for wireless multimedia sensor networks and discussed in detail the open research issues. Adamu M.Z. and al. in [12] re-simulate different routing protocols using a Matlab based simulator and give simulation results and performance metrics to serve as a benchmark for future comparison for the research community. Chee-Yee Chong and al. presents in [13] some recent research results in sensor network algorithms, including localized algorithms and directed diffusion, distributed tracking in wireless ad hoc networks, and distributed classification using local agents. K.Beydoun treated in [14] the problem of routing in wireless sensor networks and proposed a design of a hierarchical routing protocol for sensor networks based on network zones partitioning.

Routing allows information transport from source to destination through a network connection. The routing problem is to determine optimal path of the packets through the network with regard to some performance criteria such as energy consumption. The goal is to find the low investment cost which routes the nominal traffic and guarantees quality of service $(\mathrm{Q} o \mathrm{~S})$ [11].

In general, routing in sensor networks can be classified, according to structure of the network, in flat routing, and Hierarchical routing. In the flat routing, all nodes typically have the same roles and features. However, hierarchical routing is performed at several levels in the sense that the view of the network is reduced. Some nodes may play specific roles in the network to route information.

Following the method of creation and maintenance of roads in routing packets, routing protocols in wireless sensor networks can be classified into three categories: proactive, reactive and hybrid [12].

The roads in the proactive routing are calculated in advance. Each node maintains multiple routing tables by exchanging control packets between neighbors. The need to maintain and check the validity of tables routing permanently (including further information which will probably not used) is the main drawback of proactive protocols. By cons, they have the important advantage requires no delay before transmitting a packet since the road is already known. OLSR and FSR are two examples of proactive protocols.

Unlike proactive protocols, reactive protocols will calculate the route on request. However, the demand routing leads to a slow global process because of the search for roads. This type of protocol has the disadvantage of being very expensive in packet transmission in the determination of routes but has the advantage of not having to maintain information unused routing tables. AODV and DSR are two examples of reactive protocols.

The hybrid routing protocols or "mixed" combine two previous types of methods (proactive and reactive). The proactive protocol is applied in a limited scope around the source (limited number of neighbors), while the reactive protocol is applied beyond the scope (distant neighbors). This combination is achieved in order to exploit the advantages of each method and bypass their limitations. ZRP and CBRP are two examples of protocols hybrids [14].

In fact, numerous research investigations on the performance and benefit of routing protocols can improve throughput, increase reliability, reduce end to end delay, and mitigate network congestion.

\section{WVSN MODEL}

A wireless video sensors network consists of a set of autonomous nodes with a small onboard camera. In sensor networks scalar systems considering capacity omnidirectional capture, two nodes are regarded as redundant if they are close to each other. In video sensor networks, cameras have a field of view and optionally, zoom capabilities. In this case two nodes can be redundant even if they are relatively distant from each other. Sometimes multiple views are desirable to resolve ambiguities; in other situations, remote nodes can provide more usable information depending on weather conditions, for instance.

At the LIUPPA laboratory P. Congduc \& al develop a wireless video sensor networks for surveillance applications [15]. This model also addresses the management of energy, since the scarcity of this resource has a strong impact on coverage as it is not realistic that all nodes can be active simultaneously. The model proposes a based coverage approach for scheduling adaptively video nodes activity, taking into account energy considerations and objectives of the application. The proposed model is based on approach using several sets covering in order to manage redundancy fields of view of the cameras. It is based on a distributed algorithm that helps each node to organize its neighbors into non-disjoint sets, each of which being a cover set that overlaps its field of view. Then, based on the activity of its neighbors, a node decides to be active or not, without compromising its coverage area. To take into account the energy and the objectives of the application, the approach provides a multi-level model based on Bezier curves activity [15]. This approach defines two classes of application: high and low risk applications. In the first class, the application 
does not need high frame capture rate, but in the second class, the majority of nodes capture at high frame capture speed to ensure better detection.

The surveillance zone of a video node $v$ is usually represented by its field of vision. Video nodes are random positions and random directions. In $[15,16]$ the authors considered a $2 \mathrm{D}$ model that defines the field of view by the 4-tuple $v\left(\mathrm{P}, \mathrm{R}_{\mathrm{s}}, \mathrm{V}\right.$, $\alpha$ ), where $P$ represents the position, $R_{S}$ the coverage radius, $V$ the direction vector of the camera, and $\alpha$ the semi angle of view of the sensor video as illustrated in Fig 1

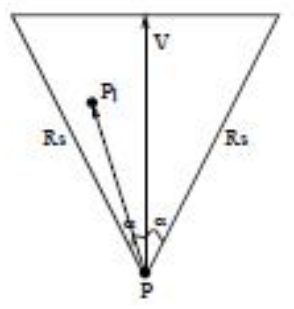

Fig 1: Video Sensing Model

First, we suppose that all nodes are identical, i.e they have the same coverage radius $R_{s}$ and the same viewing angle. The approach is fully distributed where each node must provide its own coverage independently. For the sake of simplicity, a node $v$ generally covers a triangular area with its field of vision, either by itself if it is being in an active state or by redundant nodes when it is in an inactive state. This approach allows nodes to find among their neighbors which of them cover their field of vision as illustrated in Fig 2.

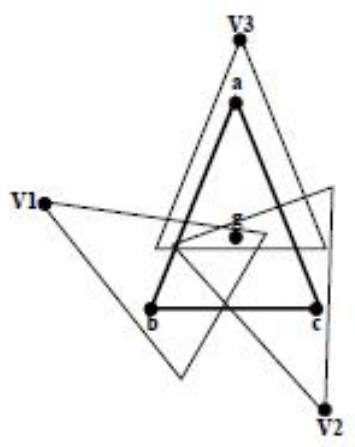

Fig 2: FoV coverage

It begins to calculate the cover set according to their cardinality, giving priority to those that contain fewer elements. In the case of two or more sets have the same cardinality; they will be classified according to their energy level. A video node $v$ receives messages of activity of its neighbors and checks if any of its cover set is satisfied or not. If a set covering $v$ is active, the node $v$ is asleep and sends its decision to its neighbors. On the other hand, if no set covering $v$ is satisfied that node $v$ decides to stay active and also broadcasts its position to its neighbors.
In video sensor networks, autonomy of a sensor is directly related to the speed of image capture. The rate of capture can be synonymous with quality of monitoring and it depends on the criticality of the application. An application designed for intrusion detection should have a fairly high speed capture to avoid missing intrusion. In other applications, the capture rate can be greatly reduced. Thus, it is important to adjust the capture rate to allow a fair compromise between autonomy, criticality and coverage. The model proposes two approaches to regulating the speed of image capture. The first approach is called static control. This is a naive approach; it is to set a constant speed of capture throughout the life of the sensor according to the criticality of the application. Its major drawback is the rapid depletion of the network. The second approach is called dynamic approach which optimizes use of energy in the network. It varies the speed of capture of a node $v$ as a function of the number of sensors they cover. In other words, the larger the area of a sensor is covered, the more it can afford to quickly capture.

\section{ALERT ROUTING ALGORITHM}

Our contribution is to extend the previous wireless video sensor networks model defined in section 3 by managing the alert diffusion. In this model, when a node detects an intrusion, it floods automatically the entire network by bypassing the message alerts to the whole network. This technique doesn't preserve energy and could create implosion effect. This effect is caused by duplicated messages sent to the same nodes. When an intrusion occurs, two nodes sensing the same region send alert packets to the same neighbors. The main idea of our algorithm is to reduce the flooding of the message alert by propagating them only for the list of the sensors located in the field of view (FoV) of the sensor alerted in such a way that only nodes able to retransmit the message in the wright direction are considered.

Figure 3 shows the operation of the noSelectiveFoV strategy. In this strategy when a node $\mathrm{v}$ detects an intrusion it spreads a message of warning to all its neighbors. Thereafter, any node receiving this message will carry an alert dissemination. Its major inconvenient is the risk of duplication of the same warning message.

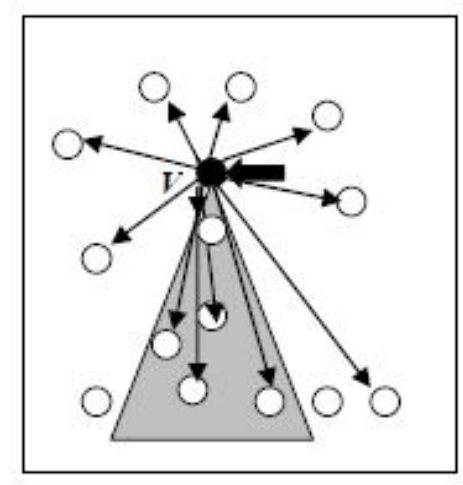


Fig 3: noSelectiveFov Alert propagation

What is more, it is disadvantageous in terms of energy consumption and obstructs the traffic in the network. In the contrary, in selectiveFoV technique, as shown in Figure 4, once the node $\mathrm{v}$ detects an intrusion it spreads a warning message to the nodes that are within its field of view. Thus, it greatly reduces the effect of this flood alert that arrives more quickly and then, increases the lifetime of the network.

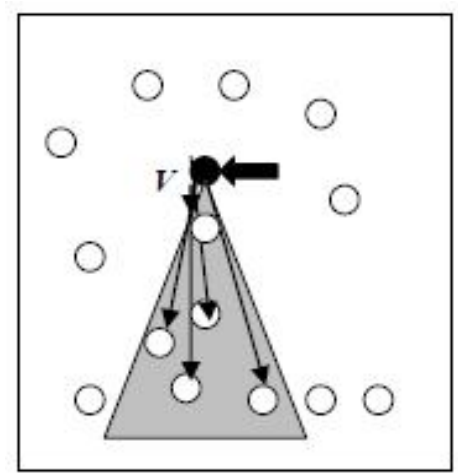

Fig 4: SelectiveFov Alert propagation

By varying the angle of view of the camera, a virtual field of vision is obtained as shown in Figure 5. The VirtualFoV strategy is based on this principle to expand the number of nodes to be alerted in the case of intrusion detection.

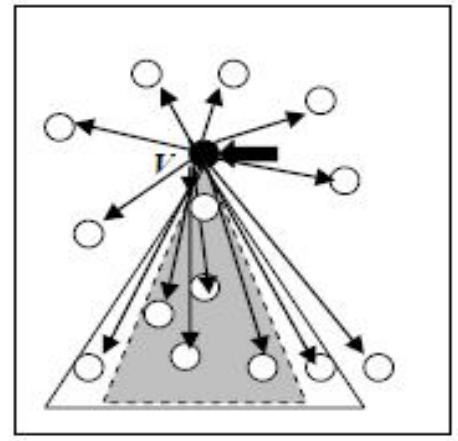

Fig 5: VirtualFov Alert propagation

So, our algorithm improves the phenomenon of diffusion in the network, and will obviously reduce energy consuming. When a node $v$ detects an intrusion it looks in its table of neighbors the list of nodes $v^{\prime}$ that are in its field of view. If this node $v^{\prime}$ is in active mode will be alerted and if it was already alerted it switches reinforcement. We shall give now, the proposed algorithms.

\section{Algorithm selectiveFOV:}

Input: intrusion detected

Output: alert packet

\section{Begin}

List :="
If node $V$ detects intrusion Then

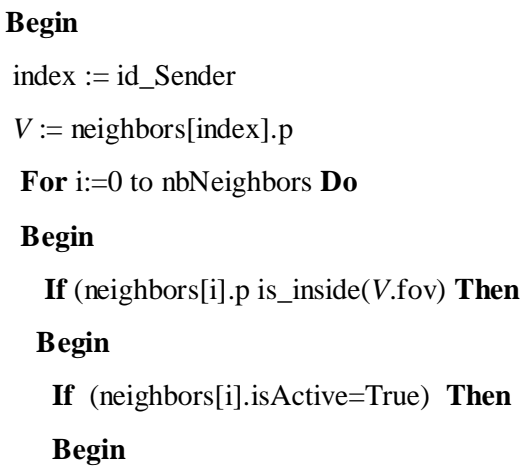

Increase AlertCriticalitylevel

SetAlertCriticalityLevel

list := list + "\#" + neighbors[i].id

End

Else

Begin

neighbors[i].status := Active

Endif

Endif

Add list to Alert packet

Send Alert packet to RoutingModule

Enddo

Endif

list_id := Packet.list

For each node $V^{\prime}$ receives Alert Packet Do

Begin

If $V^{\prime}$.id is in list_id Then

Begin

If $\left(V^{\prime}\right.$.Alerted $==$ False $)$ Then

Alerted := True

Else

Begin

Increase AlertCriticalitylevel

SetAlertCriticalityLevel

\section{Endif}

Send Alert to neighbors

Endif

Enddo

\section{End}

"noSelectiveFOV" refers to the technique used by LIUPPA [19]. It is based on the dissemination of alert after intrusion detection. It is applied for all neighboring nodes, and may causes flooding of the network. 
"selectiveFOV" method is our new technique which is based on sending the alert to the subset of neighboring nodes that are in the FOV of the node that detected the intrusion. This technique significantly reduces the propagating phenomena of sending alert and it will have a significant impact on energy consumption in the network.

"virtualFOV" is another variant of selectiveFOV where we vary the angle of view to obtain a virtual FOV.

We propose an algorithm to integrate and manage the three propagation techniques alerts in the previous model. If the severity level is at a low level implementation, we will propagate alerts based on selectiveFoV algorithm to neighbors which are in the field of view of the node that detected the intrusion. In the presence of intrusions case, there will be reinforcements alert based on virtualFoV algorithm. It will expand its field of vision by increasing the angle of view of its sensors. The alert level here is considered a medium level. It will subsequently alert a larger number of neighbors in the virtual field of vision of the node that detected the alarm. But once the alert goes to a higher level, the model uses the spread

\section{SIMULATION AND RESULTS}

To evaluate the performance of our alert routing techniques we concluded a series of simulations based on the discrete event simulators Omnetpp/Castalia (http:/www.omnetpp.org) [17] and (http:/Castalia.research.nicta.com.au) [18] under the LIUPPA wireless video sensors network model.

The LIUPPA simulation model represents a randomly deployed wireless video sensor network in an $75 \mathrm{mx} 75 \mathrm{~m}$ field. Each sensor node is characterized by: its position $(\mathrm{x}, \mathrm{y})$, a depth of view for the camera, a line of sight for the camera and an angle of view (AoV). The sensor`s field of view (FoV) is then represented by a triangle as shown in fig 1 . Depending on the number of nodes deployed in the field, the simulation will determine the cover sets for each sensor node and will compute a percentage of coverage for each cover set. Then each sensor will decide to be active or not, and will decrease its energy level according to its frame capture rate. The frame capture rate for each sensor is determined by the size of its cover sets. Also depending on the criticality level of the application, the capture rate for a given number of cover sets is varied when the initial cover set computation ends, energy node will decide to be active or not and the simulation model will determine which cover sets are active. The simulation ends when all sensor nodes have determined their cover sets and have computed for each cover set the percentage of coverage of the cover set [16]. This section aims to discuss the results of the simulation of our algorithm. Simulation was realized using Castalia environment consisting of 300 nodes. Application layer represents the videoSensorModel, Bypass Castalia in the routing layer and CSMA as Mac Layer.

These simulations are run ten times randomly generated by Castalia. The different figures below summarize our results. Fig. 6 gives an idea of the number of the sent alert packets alert based on a flood represented here by the algorithm noSelectiveFoV
Algorithm scheduling Alert:
Begin
Level := Current_CriticalityLevel;
If Level $=$ "Low" Then
Uses SelectiveFov algorithm for routing alert
Else
if Level $=$ "Medium" Then
Uses VirtualFov algorithm for routing alert
Else
Uses NoselectiveFov algorithm for routing alert
Endif
Endif

End.

issued by each technique. We note that the technique selectiveFOV has significantly reduced the number of these packets.

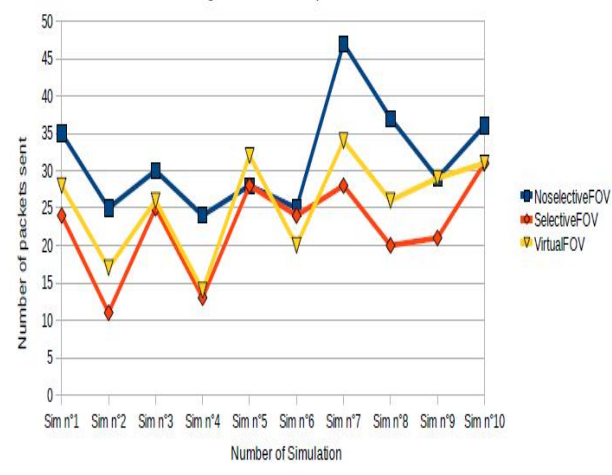

Fig 6. Number of Packet Sent

With selectiveFOV technique the number of received packets is reduced compared to the noSelectiveFOV as shown in fig 7. If we take the results of the simulation "sim $n^{\circ} 8$ ", we find that the number of received packets is equal to 150 packets with the selectiveFOV technique against 350 packets with the noSelectiveFOV. 


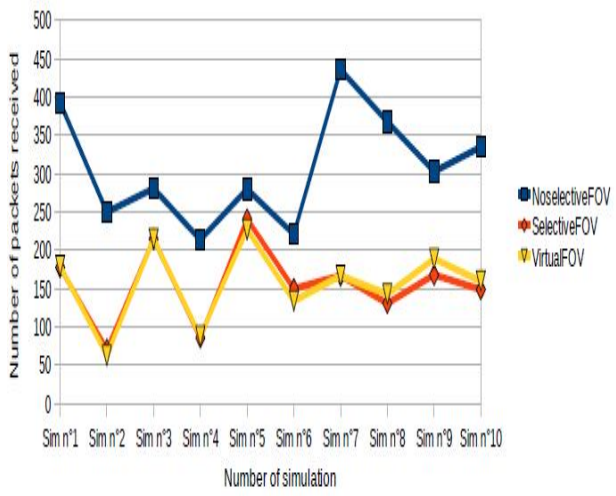

Fig 7. Number of Packet received

Fig 8 summarizes the number of detected intrusions in the network. In this case, the behavior of the three methods converges to the same values in the most phases of simulation.

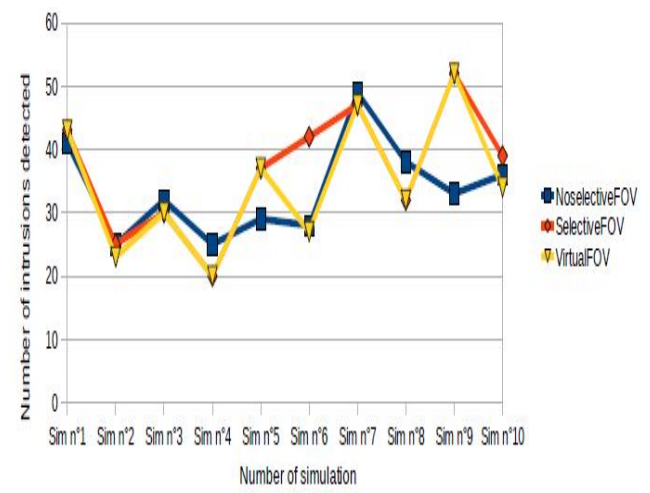

Fig 8. Number of intrusion detected

Fig 9 summarizes the difference in the energy consumption. The selectiveFOV technique gave a good preservation of the energy, and this for the various tests on the basis of the progressive variation of the running time.

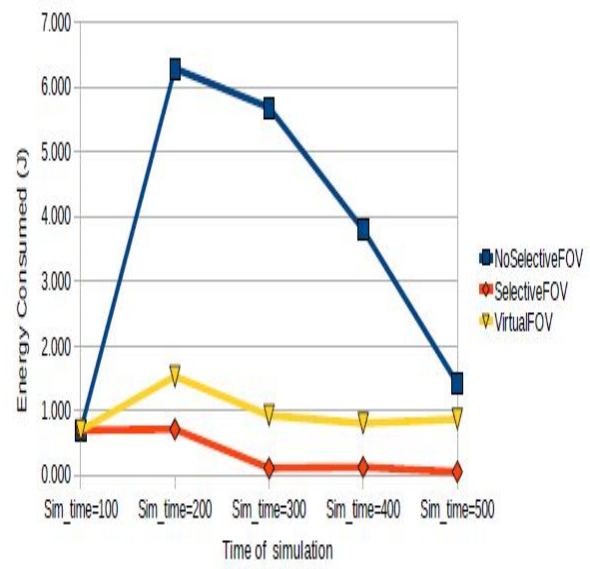

Fig 9. Energy Consumed

The simulation shows that our algorithm has given better results and then it allows good energy preservation.

\section{CONCLUSION}

This paper addresses the problem of routing alert for wireless video sensor networks deployed for critical surveillance applications. Increasing the network lifetime is of prime importance for mission-critical video sensor networks. Based on a coverage model that handles FoV concepts, we proposed a routing algorithm to manage alert propagation in the network based on a selective field of view to improve forward alerts propagation. It reduces in one hand, considerably the effects of implosion, and the number of alert packets in the network, and It takes in the other hand, into account the risk level in intrusion detection systems. Simulation results show the performance of our strategies in terms of percentage of send packets, received packets, intrusion detection and energy preservation. Future works will investigate in more details how to secure alert packets propagation in the network.

As well as security problem, the effect of the sensor failures on the Quality of Service (QoS) is an open research topic. Since sensor failures are common, fault tolerance of the network should be investigated because loss of individual sensor nodes or a group of sensor nodes should not hamper the task accomplishment of the network.

\section{REFERENCES}

[1] Pratikkumar,D. 2009. Localisation and Surveillance using Wireless Sensor Network and Pan/Tilt Camera. University Dayton, Ohio.

[2] Saaed, U. 2010. Energy and Distorsion Analysis of Video Compression Schemes in Multi-Hop Wireless Video Sensor Networks. University of Sciences and Technology Istanbul, Pakistan.

[3] Ian, F. and Kaushik, R. 2008. Wireless Multimedia Sensor Networks. Proceeding of the IEEE Vol.96, $\mathrm{N}^{\circ} 10$.

[4] Aruba networks. 2010. Improving Coverage and Quality in Video Surveillance Applications. 
[5] Terrance, E., Johnson, R., and Tracy, P. 2006. A decade of networked intelligent video surveillance. ACM Workshop on distributed camera network.

[6] Ertan, O. 1997. Deployment Quality Measures in Surveillance Wireless Sensor Networks. Thesis, Computer Engineering, Ege University.

[7] Can, C., Mehmet, Y., and Cem, E. 2012. Detection Quality of Border Surveillance Wireless Sensor Networks in The Existence of Trespassers favorite Paths. Computer Communication Journal 35 1185-1199.

[8] Antonio-Javier, G., Felipe, G., and Joan, G. 2011. Wireless Network deployment for Integrating Video Surveillance and Data Monitoring in Precision Agriculture over Distributed Corps. Computers and electronics in Agriculture Journal 75 288-303.

[9] Congduc, P., Adellah, M., and Rachid, S. 2011. Riskbased Adaptive Scheduling in Randomly Deployed Video Sensor Networks for Critical Surveillance Applications. Journal of Network and Computer.

[10] Akaya, K., Mohamed, Y. 2005. A Survey on Routing Protocols for Wireless Sensor Networks. Ad Hoc Networks 3 325-349.

[11] Ian, F.A., Tommaso, M., and Kaushik, R.C. 2007. A survey on Wireless Multimedia Sensor Networks. Computer Networks 51 921-960.
[12] Adamu, M.Z., Li-Minn, A., and Kah, P.S. 2012. Classical and Swarm Intelligence Based Routing Protocols for Wireless Sensor Networks: A survey and Comparison. Journal of Networks and Computer applications 35 1508-1536.

[13] Chee-Yee, C., and Srikanta, P.K. 2003. Sensor Networks: Evolutions, Opportunities, and Challenges. Proceeding of the IEEE, Vol.91, $\mathrm{N}^{\circ} .8$.

[14] Beydoun, K. 2009. Designing a Hierarchical Routing Protocol for Wireless Sensor Networks. PhD, University of Franche-Compte, France.

[15] Congduc, P., Abdellah, M., and Rachid, S. 2009. Scheduling Visual Sensor Nodes in Critical Surveillance Application. University of Pau, France.

[16] Congduc, P., and Abdellah, M. 2010. Performance Study of Multiple Cover-set Strategies for Mission-critical video Surveillance with wireless video Sensors. IEEE WIMBO.

[17] Omnetpp Network Simulation Framework. 2013. http//www.omnetpp.org.

[18] Castalia-NICTA. 2013. http://web.univ-pau.fr/cpham. 2013. 